\title{
The Passive Blast Protection Valve Flow Field Numerical Simulation and Movement Analysis
}

\author{
MO Caiyou ${ }^{1, a}$, ZENG Xiangwei ${ }^{2, b}$, XIANG Kefeng ${ }^{3, c}$ \\ ${ }^{1}$ Applied technology school, Southwest University of Science and Technology, Mianyang Sichuan \\ 621010, China \\ ${ }^{2,3}$ School of Manufacturing Science and Engineering, Southwest University of Science and \\ Technology, Mianyang Sichuan 621010, China \\ amocaiyou@163.com, ${ }^{\text {b kxzxw@163.com, }{ }^{c} x k f 523 @ 163 . c o m ~}$
}

Keywords: The Passive Blast Protection Valve; Pressure Drop; The Numerical Simulation; Reliability

\begin{abstract}
For the accurate analysis of flow field inside the blast protection valve occurred after blasting and the valve core movement situation, it is concluded that more accurate dynamic characteristics of flow field, using the method of computational fluid dynamics (CFD), with the aid of software Fluent6.2, for the DN50 blast protection valve blasting in blasting instantaneous flow field in the valve and valve core movement characteristics for simulation analysis. Concluded: (1) according to the flow field visualization research, blasting, blasting protection valve in the fluid velocity field, pressure field distribution is axisymmetric, effectively weaken the radial force of the fluid and the role of the valve core; (2)According to the valve core displacement, velocity and force of valve core, the outlet flow Parameters such as change over time; Instant close valve in blasting quickly, which can effectively close the valve port, verify the reliability of blasting protection valve and quick effectiveness, is conducive to the further research and application.
\end{abstract}

\section{Introduction}

In recent years, in view of the long distance pipeline leakage, blasting, Xiang-wei Zeng researchers working on "passive" for many years, puts forward a kind of the passive blast protection valve, and established a complete blast protection system, under the condition of without external force can quickly and effectively prevent further accident[1].

Because of the complexity of the pipeline flow field after blasting, the theoretical calculation is not fully express blasting under transient protection valve and pipe flow velocity, the pressure distribution, size and direction of the valve core motion law, etc. Under the experimental measurement of transient flow field in the valve and valve core movement characteristics are very difficult [2].

\section{The passive blast protection valve working principle}

The passive blast protection valve is used in pipeline system blasting moments on both ends of the valve pressure drop of drive valve core movement quickly close the valve port, to prevent further occurrence of a new type of valve leakage, can prevent the pipe leakage or blasting result in enormous outflow liquid, cause energy waste and environment pollution. Its working principle is shown in fig. 1, consists of the valve body, valve core and compression spring and so on, the normal of fluid flow, when the pipe valve core fluid impact force and spring force is in a state of relative balance, under the action of the valve under a certain opening mouth, when the downstream pipeline leakage (hole) or explosive (fracture), valve moments on both ends to create a high differential pressure, differential pressure, the function of thrust to overcome the spring force to push the valve core movement to the right, quick closing valve port. When the pipeline system after repair, valve core under the action of spring force, automatic reset and system is back to normal work $[3,4,5]$. 


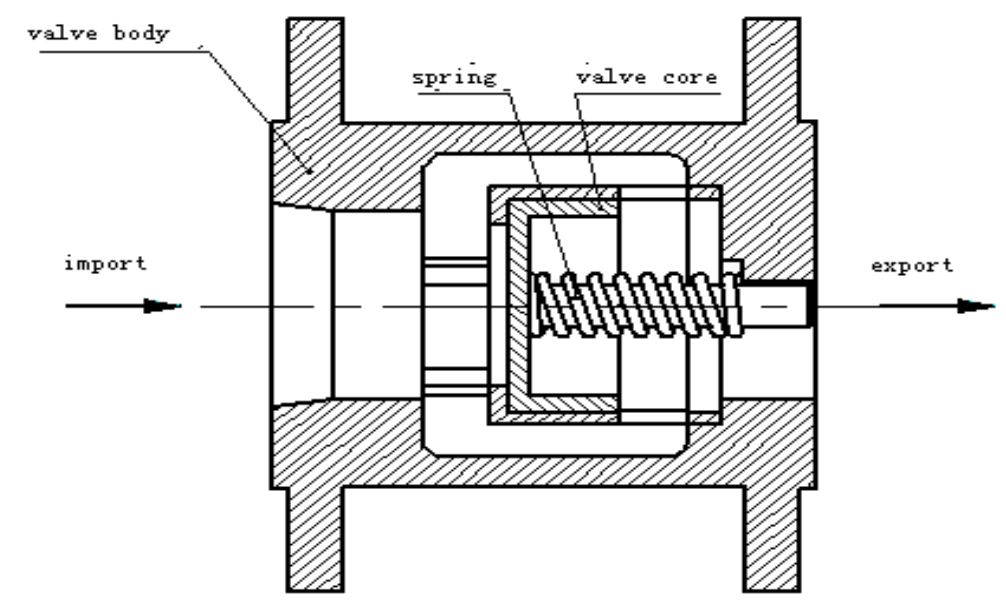

Fig. 1 The Passive Blast Protection Valve Structure Schematic Diagram

\section{Geometric modeling and meshing}

Simulate the blasting protection valve size as shown in figure 1, inlet diameter of $50 \mathrm{~mm}$, body length of $108 \mathrm{~mm}$. Considering the import period of influence on the flow field in the valve, at the inlet section of the extension is $500 \mathrm{~m}$ pipe, in the $1000 \mathrm{~mm}$ from valve outlet for pipe burst in. Due to the geometric structure of the spring flow field is affected, no spring geometry structure model is set up, but considered in the calculation of the elastic force of spring. Blasting protection valve geometry symmetry for the center, so, to simulate the two-dimensional model can be used with Fluent6.2 before processing software Gambit2.4 geometric model is set up, eventually establish geometric model shown in fig. 2. In addition, 2D model is axisymmetric structure, only calculate the half basin.

Fig. 2 The Passive Blast Protection Valve Geometry Model

To establish a good model for grid Partition, using structured quadrilateral mesh division, the area of the valve core movement grid moderate encryption, in order to make results more accurate. After dividing the local grid is shown in fig. 3. The total number of grid when the initial state in 61583, the grid independence trial, add grid almost have no effect on the result of the calculation.

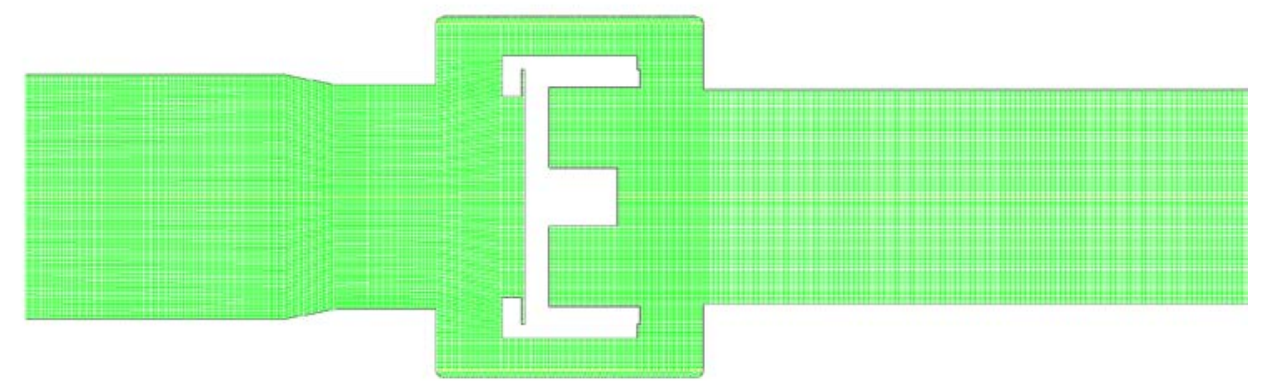

Fig. 3 The Passive Blast Protection Valve Local Grid Map

\section{Mathematical model}

\subsection{The basic control equation}

Using Euler's method simulating the flow field inside the blast protection valve, simulation of the medium for compressible gas, the continuity equation and momentum conservation equation for [6,7]:

Continuity equation:

$$
\frac{\partial \rho}{\partial t}+\frac{\partial}{\partial x}\left(\rho v_{x}\right)+\frac{\partial}{\partial r}\left(\rho v_{r}\right)+\frac{\rho v_{r}}{r}=S_{m}
$$


Momentum conservation equation, the two-dimensional axisymmetric structure, the axial and radial momentum conservation equation are:

The axial momentum conservation equation:

$$
\begin{aligned}
& \frac{\partial}{\partial t}\left(\rho v_{x}\right)+\frac{1}{r} \frac{\partial}{\partial x}\left(r \rho v_{x} v_{x}\right)+\frac{1}{r} \frac{\partial}{\partial r}\left(r \rho v_{r} v_{x}\right)=-\frac{\partial p}{\partial x}+\frac{1}{r} \frac{\partial}{\partial x}\left[r \mu\left(2 \frac{\partial v_{x}}{\partial x}-\frac{2}{3}(\nabla \cdot \vec{v})\right)\right] \\
& +\frac{1}{r} \frac{\partial}{\partial r}\left[r \mu\left(\frac{\partial v_{x}}{\partial r}+\frac{\partial v_{r}}{\partial x}\right)\right]+F_{x}
\end{aligned}
$$

Radial momentum conservation equation:

$$
\begin{aligned}
& \frac{\partial}{\partial t}\left(\rho v_{x}\right)+\frac{1}{r} \frac{\partial}{\partial x}\left(r \rho v_{x} v_{r}\right)+\frac{1}{r} \frac{\partial}{\partial r}\left(r \rho v_{r} v_{r}\right)=-\frac{\partial p}{\partial r}+\frac{1}{r} \frac{\partial}{\partial x}\left[r \mu\left(\frac{\partial v_{r}}{\partial x}+\frac{\partial v_{x}}{\partial r}\right)\right] \\
& +\frac{1}{r} \frac{\partial}{\partial r}\left[r \mu\left(2 \frac{\partial v_{r}}{\partial r}-\frac{2}{3}(\nabla \cdot \vec{v})\right)\right]-2 \mu \frac{v_{r}}{r^{2}}+\frac{2}{3} \frac{\mu}{r}(\nabla \cdot \vec{\bullet})+\rho \frac{v_{z}^{2}}{r}+F_{r}
\end{aligned}
$$

Type of $\rho$ is fluid density, using the ideal gas state equation to calculate body; $t$ is time; $v$ is the speed of the fluid. Subscript $x$ and $r$, respectively, on behalf of the axial and radial. $v_{z}$ as rotating speed, is set to zero.

In addition, in the initial conditions, the body fluid and the pipe of high Reynolds number, belongs to the turbulent state, this Paper selects the RNG k - epsilon turbulence model on the turbulent fluid in the system are described.

4.2 Valve core movement equation

Valve core is mainly by the forces of fluid pressure, viscous shear force, resistance, buoyancy, its own gravity and spring support and friction between the disc and racks. Simulation of the valve body is horizontal, so the stress of the valve core upward movement in the shaft for pressure, viscous shear force, spring resistance and friction, the friction is small can be neglected, by Newton's second law, the axial movement of the disc equation can be represented as:

$$
F_{i}=\oiint_{A}\left(p_{i}+\tau_{i}\right) d A-K\left(x-x_{0}\right)=m a=m \frac{d u_{i}}{d t}
$$

Type in the Pi and $\tau_{\mathrm{i}}$ positive pressure and viscous shear force respectively in the valve core point upward projection shaft component values. Elastic coefficient $\mathrm{K}$ for spring. $\mathrm{M}$ for the quality of the valve core. Eq.4 integral can get the displacement of the valve core in every moment:

$$
\frac{d x}{d t}=u_{i}
$$

\section{Simulation working condition}

Simulation using media as air, pressure or gauge pressure change $\mathrm{P}_{\text {in }}=1500 \mathrm{~Pa}, \mathrm{P}_{\text {out }}=$ $1400 \mathrm{~Pa}$, elasticity coefficient $\mathrm{K}=80 \mathrm{~N} / \mathrm{m}, \mathrm{t}_{\mathrm{b}}=1.0 \mathrm{~s}$. Considering the effect of pressure on the gas density, the use of the ideal gas state equation to calculate. But because the temperature change is not big, in the study no changes on the body and the pipe temperature ${ }^{[8]}$. Simulation when first set import and export pressure differential and valve core under the action of pressure difference and elastic force, open to a stable opening, then in the $1000 \mathrm{~mm}$ pipe valve port or export instant burst, burst inlet Total pressure or total pressure remains the same, but the export table pressure or the local atmospheric pressure quickly reduced to zero, to simplify the calculation, suppose in blasting instantaneously, the outlet pressure of reduced according to the linear change, as shown in fig. 4 . 


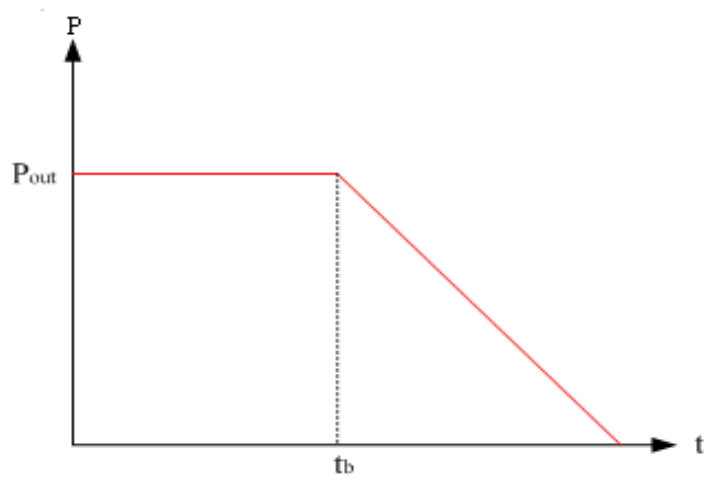

Fig. 4 Blasting Times outlet Pressure Change

\section{Calculation results and analysis}

\subsection{Velocity field changes with time}

Fig. 5 to set the blasting under the condition of instantaneous velocity field inside the valve core in the process of closing valve cloud along with the change of time. Fig. 5(a) for the zero moment, it said in a stage of stable jobs before blasting protection valve and closing phase of a on behalf of the blasting protection valve under normal working state of the flow field velocity distribution, by visible in fig. 5(a), belongs to axisymmetric structures due to blasting protection valve, the flow field velocity distribution in axisymmetric distribution, body structure is right-angle

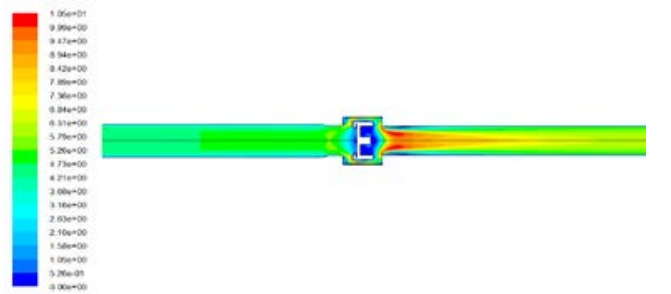

(a) $0 \mathrm{~s}$
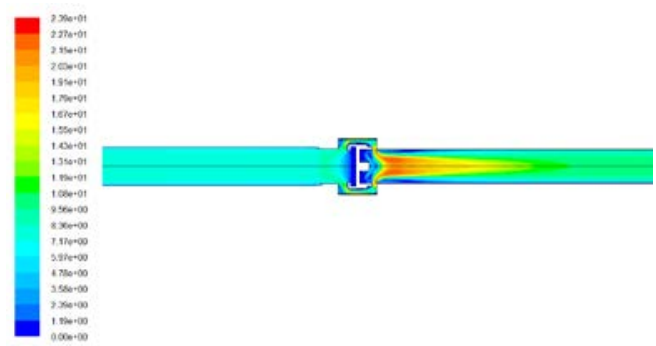

(c) $0.2 \mathrm{~s}$

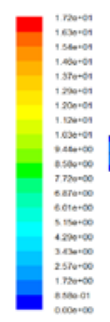

(e) $0.4 \mathrm{~s}$

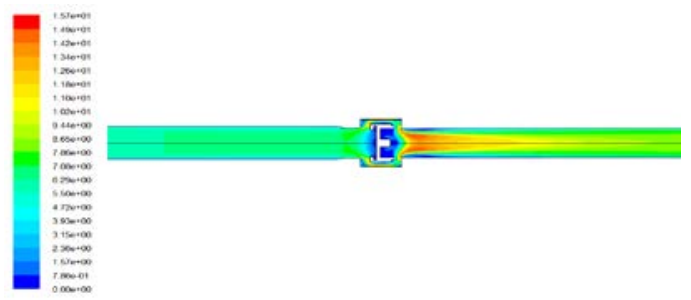

(b) $0.1 \mathrm{~s}$
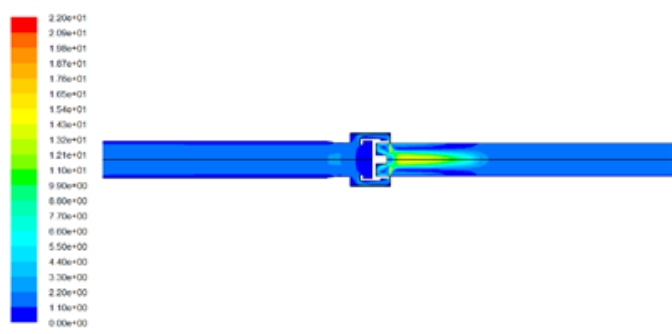

(d) $0.3 \mathrm{~s}$
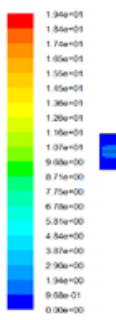

Fig. 5 Change Characteristics of Blasting Velocity Field within the Protection Valve $(K=80)$ bend forming fluid jet, fluid flow rate increased rapidly; The inner groove, because the valve core flow formed in the "dead zone".

Respectively to intercept the blasting close moments of $0.1 \mathrm{~s}, 0.2 \mathrm{~s}, 0.3 \mathrm{~s}, 0.4 \mathrm{~s}, 0.5 \mathrm{~s}$ five times velocity contours in the valve, reflect different time changes of the velocity field inside the valve. When blasting hair, outlet pressure, fluid pressure difference before and after the valve core, valve core to the right, the valve port opening gradually decreases, and the valve port at the exit of high-speed whole circulation area is reduced, but it is important to note that the valve mouth due to 
the opening decreases, and velocity changes significantly, according to the principle of fluid flow continuity, fluid must have quality, opening decreases, and the corner exit velocity must increase, and increase the impact strength of the valve and pipeline, the impact of noise enhanced accordingly.

\subsection{Pressure field change over time}

Fig. 6 for blasting moment, at various points in the process of valve closing stress nephogram. Among them, the fig.6(a) under normal working state or blasting protection valve blasting initial moment, valve pressure distribution of flow field in the cloud. Visible from the figure, the valve core is on the front of the block, there is a high end face in front of the valve core, and the pressure distribution for multi-level, the more close to the valve core end face, the higher the pressure, the valve due to the internal corner, complex pressure distribution in areas more pressure mutations, large pressure gradient and flow state is unstable, the valve core and the lining of the impact is bigger, is easy to form the impact noise.

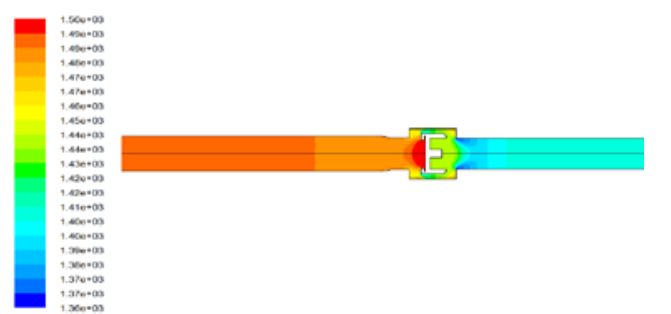

(a) $0 \mathrm{~s}$

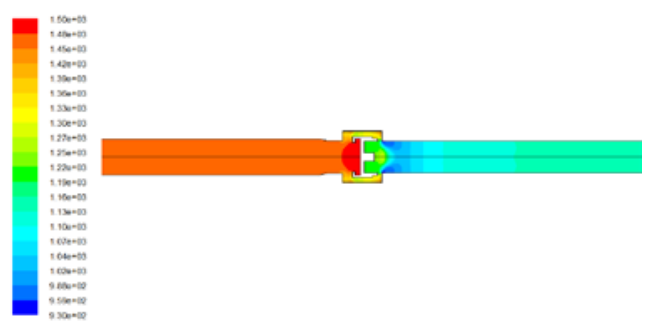

(c) $0.2 \mathrm{~s}$

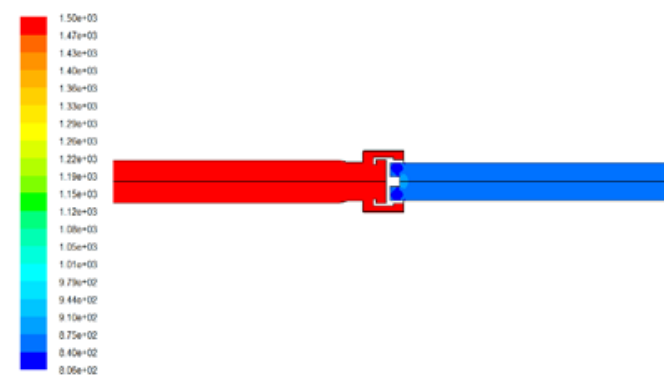

(e) $0.4 \mathrm{~s}$

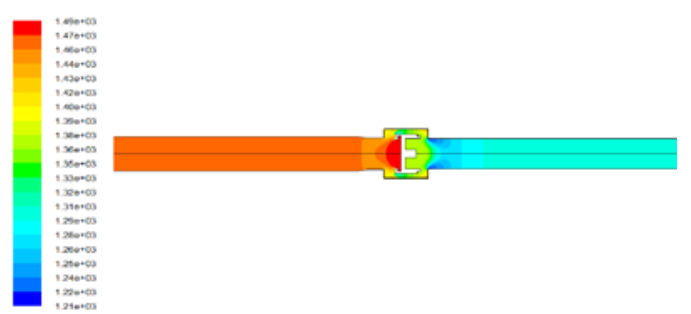

(b) $0.1 \mathrm{~s}$

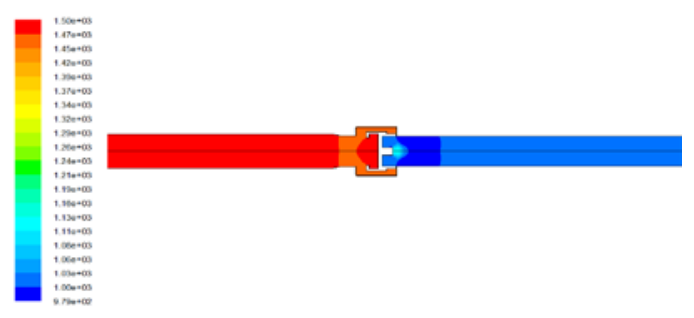

(d) $0.3 \mathrm{~s}$

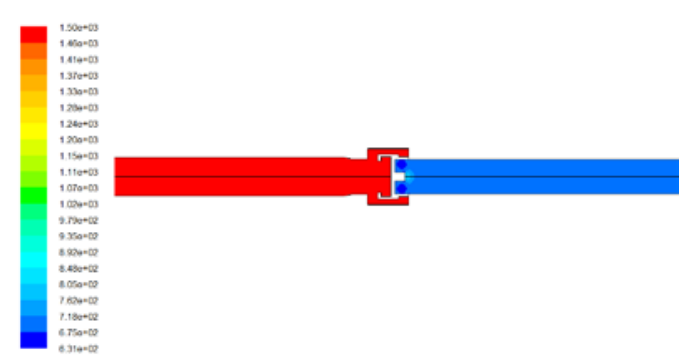

(f) $0.5 \mathrm{~s}$

Fig. 6 Change Characteristics of Blasting Stress Field Inside the Protection Valve $(K=80)$

As pipe blasting, driven by the pressure difference, the valve core to exit move, valve opening mouth constantly decreases, and continue to hinder fluid flow, increasing pressure on the valve front end to the inlet pressure, the outlet pressure is gradually reduced to the local atmospheric pressure, gauge pressure reduced to zero. Before and after the valve core pressure difference is very big, have played an important role in preventing fluid through the valve core, to achieve the effect of the blasting protection valve.

Fig. 7 for blasting moment in the process of valve closing, import and export of pipeline static pressure along with the change of time curve. Simulation of the working conditions for the Pin=1500Pa, Pout $=1400 \mathrm{~Pa}, \mathrm{~K}=80 \mathrm{n} / \mathrm{m}$, blasting moment in $\mathrm{t}_{\mathrm{b}}=1.0 \mathrm{~s}$. $0 \sim 1.0 \mathrm{~s}$, for the system to work normally. Due to set the inlet pressure of 1500Pa for fluid of total pressure, total pressure consists of two parts, static pressure and dynamic pressure, when blasting protection valve to gradually open, fluid velocity increase, Part of the dynamic pressure, static pressure is gradually transformed into imports of static pressure reduced to $1490 \mathrm{~Pa}$ from the initial conditions of $1500 \mathrm{~Pa}$ and stable; Begin to 1.0s, blasting, outlet pressure within 1.0s linear decrease to zero, and the inlet 
pressure with the valve on both ends of the transient pressure difference is bigger, gas flow rate increases, lead to rapid of fluid flow on both ends of the valve, static to dynamic pressure rapidly, within about $0.2 \mathrm{~s}$ after blasting import pressure fast attenuation to the inlet side of the lowest, 1472Pa. As blasting, the opening of the valve core decreases, the flow of gas to reduce gradually, pipe gas velocity decreases, and the pressure decreases, the pressure gradually to static pressure, so after the pressure drop to the lowest, static pressure recovery gradually, when the valve core and valve body contact moment, collisions result in valve core rebound, causes the inlet pressure to produce slight fluctuations, but becoming more stable.

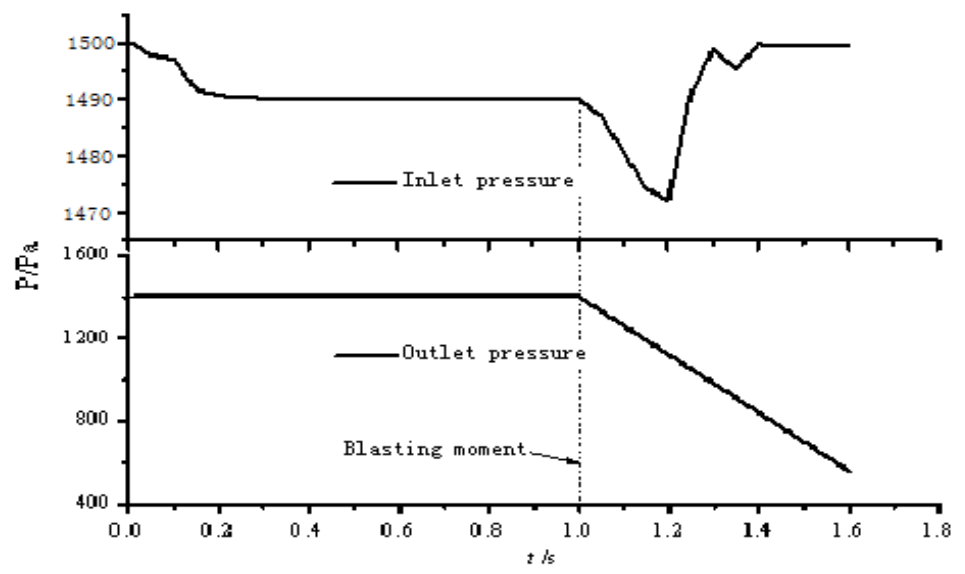

Fig. 7 In Blasting Import and Export Pressure Changing with time Curve $\quad\left(\mathrm{P}_{\text {out }}=1400 \mathrm{~Pa}\right.$, $\left.\mathrm{K}=80 \mathrm{~N} / \mathrm{m}, \mathrm{t}_{\mathrm{b}}=1.0 \mathrm{~s}\right)$

\subsection{Pipe outlet flow changes over time}

The valve outlet flow changes over time is an important feature of reflecting the explosion protection valve performance Parameters. The fig. 8 for the export flow curve of change over time. As you can see from figure, when the valve is open, flow rate increases to a stable value, under the conditions of normal work flow value is about $0.01 \mathrm{Kg} / \mathrm{s}$, blasting, due to the differential pressure transient increase on both ends of the valve, outlet flow increase rapidly, maximum flow rate of $0.0165 \mathrm{Kg} / \mathrm{s}$, and the static pressure corresponding to the above, the time is about $0.2 \mathrm{~s}$ after blasting. Move when the valve core and valve opening gradually decreases, and lead to a sharp drop in export flow, moving in the valve core to maximum displacement, as a result of the action of spring force, valve core rebound, to regain some opening valve port, a modest traffic, but under the effect of the inlet pressure, back stretch gradually disappear, because of the spring and the effect of inlet pressure, the valve can't once is closed, the valve core in the shaft up will appear many times, but over time, amplitude attenuation, valve mouth opening smaller and smaller, finally negligible, until the valve fully closed, the outlet flow is zero.

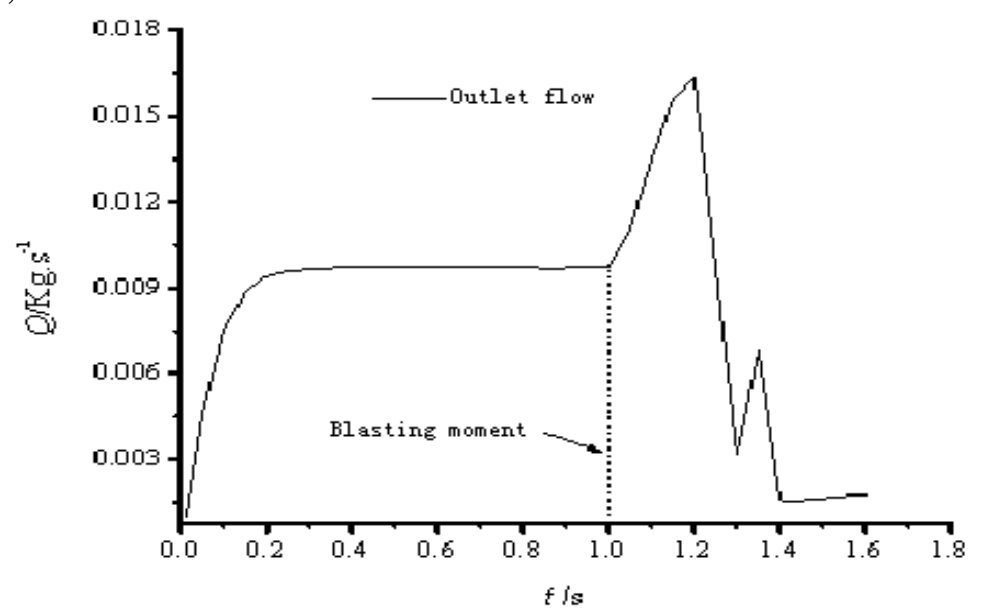

Fig. 8 Blasting Moment outlet Flow Changes over time $\left(\mathrm{P}_{\text {out }}=1400 \mathrm{~Pa}, \mathrm{~K}=80 \mathrm{~N} / \mathrm{m}, \mathrm{t}_{\mathrm{b}}=1.0 \mathrm{~s}\right)$ 6.4 Valve core fluid force and movement characteristics 
For fluid flow in complicated cases in the valve, the valve core is also affected by gravity and spring factors, stress is more complex, but because of the valve belong to axisymmetric structure model, we ignore the weight of valve core and based on the analysis of fluid on the valve core is the influence of the radial force, only consider the valve core in the role of axial force, fig.9 for blasting stress of valve core and valve movement characteristic diagram. Normal work, force balancing valve core, and resultant force is close to zero, or near zero. The valve core by the fluid force through the spring deformation calculation, or import and export pressure difference estimation, the value is very small, so the valve opening is bigger also, pipeline system can work normally. When blasting occurs, the transient pressure difference increases suddenly, the valve core force equilibrium is broken and increased. As shown in fig.9, the valve core fluid forces rapidly rising to a maximum and then decreases, then increases gradually, until completely closed, the fluid forces for the pipeline valve core import and export pressure differential and valve core axial force bearing area, the product of again in a stable state or closed. Need to point out that in the process of closing, the valve core appears the fluid pressure is the cause of a maximum and then decreased, when quick closing valve core, because of the large flow velocity in the back of the valve core section of the pressure inside the area is very low, see fig.6(d), led to pressure difference before and after the great, and the valve core fluid force to reach maximum. After reflow, see fig.6(e), the back pressure valve core rising rapidly, differential pressure decreases, and by the fluid pressure reducing valve core, the circumfluence disappear, but the valve opening, the pressure difference before and after the valve core and under the condition of the valve opening decreases gradually increased.

Due to the blasting force in an instant, the valve core motion is complex, need to analyze the movement rule of valve core. Normal working stage, due to the import and export pressure difference between the spring and are in a state of relative balance, lead to the normal work of the valve core when a periodic oscillation, amplitude and differential pressure and the spring stiffness is directly related to import and export; Blasting, the attenuation, outlet differential pressure increase on both ends of the valve core, push the valve core to move toward the outlet, it reach maximum speed,or valve fully open degree, valve spool speed from the initial oscillation value zero,almost increases rapidly to a maximum of $0.15 \mathrm{~m} / \mathrm{s}$, but with the valve opening is reduced, increased amount of spring compression, spring force increases, positive thrust to overcome the pressure difference of valve core, valve core movement speed reduced gradually, the valve core and valve mouth collision, valve opening is zero, the valve core to stop movement, from the valve core movement speed curve, blasting valve closing time about $0.4 \mathrm{~s}$, which is under the working condition, the closing of the valve spool speed faster than blasting, blasting effectiveness and fast response characteristics of the protection valve visible.

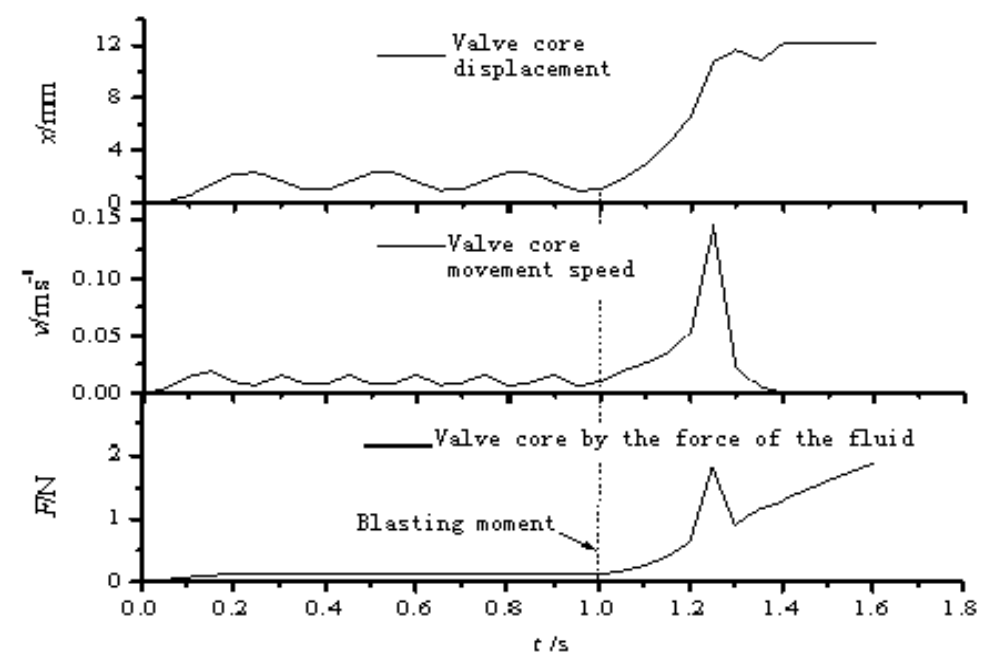

Figure 9 Blasting Force and Movement Characteristics of Valve Core $\left(\mathrm{P}_{\text {out }}=1400 \mathrm{~Pa}\right.$,

$$
\left.\mathrm{K}=80 \mathrm{~N} / \mathrm{m}, \mathrm{t}_{\mathrm{b}}=1.0 \mathrm{~s}\right)
$$




\section{Conclusion}

Through the above simulation of flow field inside the blast protection valve and flow, the dynamic visualization of the blasting process protection valve and the flow of the fluid in the pipeline, the axisymmetric flow field distribution in the valve, fluid on the radial forces cancel each other out, reduce the valve core in the process of closing up on radial move; Blasting, quickly close the valve core and valve fluid flow rapidly reduced to zero, to prevent further flow of fluid in the pipe, fully closed valve port, the effective implementation of explosion protection valve function. Is verified by numerical simulation method in this Paper, the design of the blasting protection valve the reliability and validity quickly, provide certain theoretical basis for study of experiment and data reference.

\section{Reference}

[1] Zeng Xiang-wei.Shuttle-type rupturing protection devices of pressure piping by Passive control.China, 1419640[D].2004-01-15.

[2] Zeng Xiang-wei „Huang Shou-yi,Gao Shu-fan,etal.The dynamic characteristics of Shuttle-type pipeline rupturing protection devices[J]. Oil and gas storage and transportation, 1999,18(1):30-34 .

[3] Xiang Ke-feng ,Zeng Xiang-Wei,Xiang Bei-ping.Non active pipeline rupture protection technology [J]. Journal of oil and gas field surface engineering.2012,31(7):35-36.

[4] Zeng Xiang-wei, Chen Kun, Qiu Xiao-ping, eta1.The burst protectors for the pressure pipeline in shuttle-type Passive control system[C]//Proc.9th Confer- ence on Systemics, Cybernetics and Informatics, 2005;(2):403-407.

[5] Zeng Xiang-wei, A Novel Gas/hydraulic Speed Regulating System[C]: The Application of the Differential Flow Adjustable Shuttle Valve in Speed Regulating System, Process Automation Instrumentation, Vol. 23.1:38-41.

[6] Chen Kun, Zeng Xiang-wei,Chen Wei.Finite element emulation analysis on Passively controlled burst protection system of pressure piping[A].Pressure pipeline collection technology research progress--The fourth national conference on pipeline technology[C],2010.

[7] Zeng Xiang-wei, Chen Kun, Qiu Xiao-ping, eta1.The burst protectors for the pressure pipeline in shuttle-type Passive control system[C]//Proc.9th Confer- ence on Systemics, Cybernetics and Informatics, 2005;(2):403-407. 